\title{
Chile como oferente de cooperación Sur-Sur: la progresiva adaptación a los nuevos tiempos y responsabilidades ${ }^{*}$
}

\author{
Chile as a provider of South-South \\ cooperation: the progressive adaptation \\ to new times and responsibilities
}

\author{
Guillermo Santander Campos ${ }^{* *}$
}

\begin{abstract}
RESUMEN
El presente artículo analiza la cooperación que Chile ha ofrecido a otros países de similar o menor nivel de desarrollo desde hace más de dos décadas. Para ello, se somete a estudio la política chilena de cooperación Sur-Sur, presentando los principales rasgos y particularidades de un modelo que, además de promover el desarrollo de los países de su entorno regional, conforma una respuesta adaptativa a los cambios que se están registrando en el escenario internacional en los últimos años. Conocer las implicaciones, tendencias y desafíos futuros que presenta esta política, atendiendo tanto a los factores estructurales como socio-cognitivos que inciden en ella, constituye el propósito principal de este artículo.
\end{abstract}

Palabras clave: Cooperación chilena - Cooperación Sur-Sur Sistema de cooperación para el desarrollo-Cooperación triangular.

* El artículo que aquí se presenta se deriva de la Tesis Doctoral sobre cooperación Sur-Sur defendida por el autor en la Universidad Complutense de Madrid (Santander, 20I6) y dirigida por José Antonio Alonso y Rafael Díaz-Salazar, a quienes el autor agradece sus aportaciones y permanente apoyo.

** Doctor en Ciencias Políticas y Relaciones Internacionales e investigador del Instituto Complutense de Estudios Internacionales (ICEI).guillermosantander@icei.ucm.es.

Recibido: II de enero de 20I7. Aceptado: Io de mayo de 2018. 


\section{ABSTRACT}

This article analyzes the cooperation that Chile has offered to other countries with similar or lower level of development during the last two decades. For that purpose, is analyzed the chilean policy of South-South cooperation, presenting the main features of a model that, in addition to promoting the development of countries in its regional context, it is an adaptive response to the changes that are occurring on the international system in the last years. Knowing the implications, trends and future challenges of this policy, paying attention to structural and sociocognitive factors that explain the model adopted, are the main purposes of this article.

Keywords: Chilean cooperation - South-South Cooperation Development Cooperation System - Triangular Cooperation. 
Guillermo Santander Campos

Chile como oferente de cooperación Sur-Sur: la progresiva adaptación a los nuevos tiempos y responsabilidades

\section{INTRODUCCIÓN}

Uno de los fenómenos más relevantes experimentados por el sistema internacional en los últimos años es el mayor dinamismo y protagonismo adquirido por la denominada cooperación Sur-Sur, a través de la que un país tradicionalmente considerado "en desarrollo" comparte sus recursos y capacidades con otro país de similar o menor nivel de ingreso. Se trata de un fenómeno que, además de estar en estrecha relación con los cambios y transformaciones más profundas que se están produciendo a escala global - lo que descarta su presencia meramente coyuntural o pasajera-, denota la progresiva dilución que se está produciendo en la rígida separación que tradicionalmente ha operado en el sistema internacional de cooperación (Alonso et al., 20I4; Sanahuja, 20I3 y 2008). Así, frente al clásico y reduccionista binomio "países donantes" (del Norte) / "países receptores” (del Sur), en la actualidad se asiste a un entramado más complejo y multidireccional que, como se ha señalado en algunos foros internacionales, parece demandar la conformación de un sistema de "responsabilidades compartidas, aunque diferenciadas", entre los distintos países, en lo que se refiere a la promoción del desarrollo a escala global y, de manera más amplia, a la propia gobernanza del sistema internacional.

Dentro de este contexto, Chile ha ido asumiendo de manera progresiva el desempeño de un rol dual, especialmente desde los inicios de la década de los noventa del pasado siglo. De tal modo que, sin renunciar a seguir recibiendo cooperación tradicional en determinados ámbitos, ha puesto sus capacidades y experiencias a disposición de otros países en desarrollo, erigiéndose como uno de los principales oferentes de cooperación Sur-Sur en América Latina, como destacan de forma sistemática los diversos informes existentes en la materia (SEGIB, 200820I5; CAD-OCDE, 20IO).

A partir de los resultados obtenidos en un amplio trabajo de campo —que incluyó la realización de entrevistas en profundidad con expertos y expertas, representantes de la sociedad civil y personas vinculadas a la gestión y a la toma de decisiones de la cooperación chilena ${ }^{1}$ - este artículo se dedica a presentar los principales rasgos y lógicas que caracterizan al modelo chileno de cooperación Sur-Sur, así como a analizar los principales desafíos e implicaciones que se derivan de esta política.

1 En este sentido, el autor agradece muy sinceramente su tiempo y colaboración a todas estas personas y, de manera especial, a Cristina Lazo y Paz Milet, por su apoyo y amabilidad a lo largo de un proceso que se desarrolló en el marco de una estancia de investigación en el Instituto de Estudios Internacionales (IEI) de la Universidad de Chile, institución a la que se hace extensivo el agradecimiento. 
Con ese fin, y tras esta breve introducción, el segundo epígrafe se dedica a presentar los principales elementos del marco estratégico más amplio en el que se ubica la política de cooperación de Chile. A partir de la presentación de las principales cifras disponibles, el tercer epígrafe realiza una caracterización de la cooperación Sur-Sur desplegada por Chile hasta la fecha. El cuarto epígrafe, desde un punto de vista más cualitativo y analítico, somete a estu- dio los principales rasgos que parecen definir este modelo.

El quinto epígrafe aborda el papel desempeñado por una serie de elementos de tipo socio-cognitivo que, junto con otros factores de carácter estructural, habrían condicionado la fisonomía de la cooperación chilena. Por último, en el sexto epígrafe se recoge un conjunto de conclusiones que se derivan del análisis precedente.

\section{El MARCO ESTRATÉGICO: INSERCIÓN INTERNACIONAL Y POLÍTICA EXTERIOR DE CHILE}

\section{La inserción internacional de \\ Chile: la relevancia de la apertura comercial}

Desde el punto de vista estratégico, la cooperación Sur-Sur chilena se ubica en el marco más amplio de su política exterior, una realidad que, a su vez, no puede ser entendida sin atender previamente al particular proceso de inserción internacional experimentado por el país en las últimas décadas y cuyos principales rasgos conviene señalar brevemente aquí. En este sentido, durante la dictadura de Augusto Pinochet (1973-1990), y particularmente en la década de los ochenta del pasado siglo, Chile mostró una suerte de "disociación” en su inserción internacional (Ross, 20I2): mientras en el ámbito político, el país sufría un marcado aislamiento internacional como resultado del rechazo de la comunidad internacional al régimen vigente, en el ámbito económico mostraba un elevado grado de apertura comercial hacia el resto del mundo, lo que era reconocido y puesto como ejemplo por una parte importante de esa comunidad internacional.

No obstante, con el retorno de la democracia, en 1990, este modelo de inserción económica externa fue percibido por amplios sectores como un importante logro sobre el que debía asentarse la estrategia de desarrollo del país (Quezada, 20I2). A partir de este diagnóstico compartido, y con algunas ligeras diferencias, los diversos gobiernos que desde entonces se fueron sucediendo, optaron por consolidar y profundizar un modelo de inserción económica fuertemente asentado en una amplia desregulación y liberalización comercial - con la firma de 
Chile como oferente de cooperación Sur-Sur: la progresiva adaptación a los nuevos tiempos y responsabilidades

importantes Acuerdos y Tratados de Libre Comercio- y en la atracción de Inversión Extranjera Directa (IED), de tal forma que se constituyó en un elemento común y apenas rebatido de la política exterior desplegada por todos ellos $^{2}$. La firma de un elevado número de acuerdos comerciales a lo largo de estos años, con una considerable diversificación geográfica, el peso de los flujos comerciales y de la entrada de la IED en su PIB ${ }^{3}$ o la incorporación a la OCDE, en 20I0, son expresiones de la decidida apuesta de Chile por mostrarse al mundo como una economía "flexible y abierta". Este rasgo ha constituido un pilar esencial de una política exterior que, asentada en estos instrumentos, sin duda ha mostrado más elementos de continuidad que de cambio hasta la fecha, conformando, en la práctica, una política de Estado (Aranda y Riquelme, 20II; Milet, 20I2).

Esta decidida apuesta realizada por Chile por el modelo de inserción internacional descrito, ha configurado un tipo de política exterior cuyos principales rasgos e implicaciones permean también a su propio modelo de cooperación Sur-Sur, como se verá más ade-

2 Estos gobiernos han sido los presididos por Patricio Aylwin (1990-1994), Eduardo Frei Ruiz-Tagle (1994-2000), Ricardo Lagos (2000-2006), Michelle Bachelet (200620I0), Sebastián Piñera (20I0-20I4) y, de nuevo, Michelle Bachelet (20I4-20I8).

3 Mientras en 2012 el comercio y la IED suponían, respectivamente, el 45,8\% y el 3,I\% del pIB para el conjunto de América Latina, en el caso de Chile estas cifras se elevan hasta el 68,I\% y el II,3\% (PNUD, 20I4). lante. Dos de estos rasgos convienen ser destacados aquí. Por un lado, se ha otorgado una cierta primacía a lo comercial por sobre lo político, en el posicionamiento internacional y en las relaciones exteriores desplegadas por Chile. Así, el predominio de esta visión de "política exterior comercial" habría estado en la base de la ausencia de una estrategia de política internacional que trascienda el objetivo de la inserción económica y, con ello, ha alentado un comportamiento fundamentalmente reactivo (Flisfisch, 20I2; Fermandois, 20I2) frente a un sistema internacional crecientemente complejo y cambiante, que demanda mayores dosis de visión estratégica. Por otro lado, la apuesta de Chile por este modelo de inserción económica internacional ha provocado un cierto déficit en lo que se refiere a su política exterior hacia América Latina y le ha generado un distanciamiento político con su entorno regional (Fermandois, 2006). En un contexto político, especialmente desde mediados de la década de 2000 , en el que en la región comenzaron a proliferar iniciativas de integración regional de corte "postliberal", que tratan de otorgar mayor peso y centralidad a los componentes políticos y sociales, Chile se erigía como un exponente del denominado "regionalismo abierto" que, como se vio y en sintonía con su modelo de inserción internacional, pone el énfasis en la apertura comercial y la integración fundamentalmente económica (Briceño, 20I4; Sanahuja, 2009). Así, paradójicamente, este modelo de 
política exterior pareció integrar a Chile en el mundo, a la vez que lo alejó de sus vecinos (Ross, 20I2).

Esta disonancia obligó a Chile a mantener delicados equilibrios en la región, desarrollando una "estrategia de contención y contraposición” (Flisfisch, 20I2: 546), de tal modo que se mantenía al margen o contrario a aquellas iniciativas que consideraba incompatibles con su modelo de inserción económica internacional - fundamentalmente el ALBA, pero también de otras como Mer$\operatorname{cosur}^{4}$ o la Comunidad Andina ${ }^{5}$ - a la vez que trataba de impulsar otras iniciativas subregionales que consideraba más acordes con su visión y objetivos, tales como la Alianza del Pacífico, de carácter más comercial (Prado, 20I4). Se trataba, en definitiva, de intentar profundizar la integración regional, pero hacerlo de manera compatible con la liberalización comercial (Aranda y Riquelme, 20II; Van Klaveren, 1998). Un difícil equilibrio al que se le trata de encontrar mejor acomodo en UNASUR y CELAC, iniciativas de las que Chile forma parte desde su fundación.

\section{Los fundamentos de la política exterior chilena: principios y objetivos $^{6}$}

Esta tensión entre la apuesta por profundizar una estrategia de desarrollo y un modelo de inserción económica internacional "flexible y abierto al mundo", por un lado, y el intento de reducir el distanciamiento político que en ocasiones le ha generado en su entorno regional, por otro, constituye el marco de referencia sobre el que Chile ha tratado de definir sus principios y objetivos de política exterior. Junto con el respeto al derecho internacional y la promoción de la democracia y los derechos humanos, Chile señala como uno de sus tres principios fundamentales en materia de política exterior la "responsabilidad de cooperar", entendiendo que es necesario acudir a marcos mancomunados y dar respuestas cooperativas a los desafíos que plantea la globalización. Así, asumiendo la responsabilidad que en esta tarea le corresponde como miembro de la comunidad internacional, Chile señala su "compromiso firme de cooperar, a través de sus recursos técnicos y humanos" tanto a escala bilateral, como multilateral y regional?

Como reflejo de la constante anteriormente descrita, si se atiende al decálogo de objetivos que Chile atri-

\footnotetext{
6 Este apartado es una revisión ampliada y actualizada de lo recogido en Santander (2013).

7 Información disponible en www.minrel. gob.cl
} 
buye a su política exterior, se observa que los dos primeros en aparecer son la promoción de los intereses económicos del país y la "asociación comercial con otros países", por un lado, y la contribución al "fortalecimiento de la integración regional", por otro. En lo que se refiere al primero de ellos, se enfatiza la fuerte dependencia que tiene el desarrollo del país con el comercio exterior, entendiéndose que la política exterior constituye un instrumento cada vez más relevante para "favorecer la internacionalización de nuestra economía, fortalecer la promoción comercial y fomentar el desarrollo económico y social de la nación". De ahí que se considere de "fundamental importancia" la existencia, a nivel internacional, de escenarios económicos que favorezcan el libre comercio y la reducción de barreras comerciales "de cualquier índole". Como resultado de ello, la negociación de acuerdos comerciales, la promoción de las exportaciones, la atracción de inversiones y la participación en los foros competentes en la materia, aparecen como las principales líneas de acción dentro de este primer objetivo ${ }^{8}$. Sin embargo, respecto del segundo de los objetivos señalados, existe una menor precisión en cuanto a líneas de acción, limitándose a destacar la importancia de "incrementar la cooperación, asociación y complementariedad" entre las na-

8 Información disponible en www.minrel. gob.cl ciones de la región, para potenciar los beneficios del proceso de globalización. En este sentido, destaca la importancia del desarrollo de proyectos conjuntos en áreas como la infraestructura regional y energética, la conectividad, la coordinación de políticas sociales, la pobreza, el diálogo político o el libre comercio, que son los que darían "un sentido real a la idea de integración"

Completan el decálogo de objetivos de la política exterior chilena otras ocho metas que, por su naturaleza, cabría dividir en tres grupos. Por un lado, aparecen dos objetivos que se relacionan con la gobernanza de la globalización y con la asunción de responsabilidades en el ámbito internacional, como son los que aluden al fortalecimiento del multilateralismo y la promoción de la paz y la seguridad internacional. Por otro lado, aparecen otros dos objetivos más ligados a mejorar la percepción de Chile en el exterior, como son los que se refieren al fortalecimiento de la imagen del país y a la promoción de la cultura chilena. Por último, aparecen otros cuatro objetivos que cabría vincular a intereses directos de Chile en ámbitos muy específicos, como es el caso de los que aluden a la promoción de los intereses marítimos y antárticos del país, la búsqueda de seguridad energética, su inserción en redes de ciencia y tecnolo-

9 Información disponible en www.minrel. gob.cl 
gía o, en fin, la asistencia y protección consular a los chilenos en el exterior ${ }^{10}$.

Ante la ausencia de un documento estratégico específico, estos "fundamentos de la política exterior de Chile" se limitan, pues, a trazar un amplio conjunto de principios y objetivos cuyo desarrollo y precisión tampoco son acometidos en los apartados dedicados a las relaciones exteriores del país en los sucesivos Programas de Gobierno. No obstante, en estos documentos se recogen algunas orientaciones generales que resultan de interés destacar, para completar el marco estratégico de la cooperación chilena. En el caso del Programa de Gobierno de Sebastián Piñera (2010-20I4), se establecía la "priorización de la relación con América Latina y nuestros países vecinos" (Gobierno de Chile, 20ro). Una orientación geográfica de la política exterior chilena que identificaba también ciertos ámbitos clave de trabajo, entre los que destacaban algunos especialmente relevantes para la cooperación para el desarrollo que Chile pretende impulsar, como son la modernización política, la lucha contra la pobreza, el medio ambiente o la pretensión explícita de convertirse en un centro neurálgico en materia de, entre otros aspectos, formación de capital humano en la región. Junto a ello, en este documento se identificaban otros dos ejes de acción con implicaciones en materia de cooperación para

10 Información disponible en www.minrel. gob.cl el desarrollo. Por un lado, el fortalecimiento de las relaciones con Estados Unidos y Europa, en el que, en el caso específico del primero, se señala como una prioridad el establecimiento de programas de cooperación conjunta para América Latina. Por otro lado, la transformación del Ministerio de Relaciones Exteriores "en un organismo más moderno, ágil y profesional" (Gobierno de Chile, 20Io), proceso del que no estaría exenta la AGCID.

Por su parte, el Programa de Gobierno de Michelle Bachelet (20I420I8) señala que la política exterior de Chile "debe contribuir a lograr una mayor unidad regional", fundamentalmente con América del Sur, destacándose el rol que pueden desempeñar organismos como UNASUR y CELAC. En este marco, también se valoran positivamente "los esfuerzos de integración de la Alianza del Pacífico", incidiendo en la necesidad de que no constituya una iniciativa "excluyente o antagónica" con otros procesos regionales. En todo caso, se percibe un cierto revisionismo, pendiente de contrastar en la práctica, en la medida en que a continuación se señala que "Chile ha perdido presencia en la región (...), se ha impuesto una visión mercantil de nuestros vínculos latinoamericanos y se han ideologizado las opciones de inserción externa" (Gobierno de Chile, 20I4). Una revisión que debiera tener su correlato en el fortalecimiento de la política de cooperación 
Sur-Sur desarrollada por Chile en los próximos años.

En suma, la cooperación Sur-Sur de Chile no puede comprenderse si no es en el marco más amplio de su política exterior y de las prioridades y objetivos definidos por el país en este ámbito. Un contexto dentro del cual, como se verá más adelante, por primera vez se ha elaborado un documento estratégico específico en materia de cooperación para el desarrollo.

\section{Caracterización de la cooperación Sur-Sur de Chile: ALGUNAS CIFRAS BÁSICAS ${ }^{11}$}

Uno de los aportes más relevantes realizados por la AGCID desde hace varios años ha sido la elaboración y publicación de informes sobre los flujos de cooperación ejecutados por esta entidad, documentos que se constituyen como la única fuente de información estadística relativa a la cooperación Sur-Sur de Chile. Si bien no cabe reducir la cooperación chilena a los datos de ejecución de la AGCID - por la diversidad de actores públicos implicados en esta materia y las limitaciones que AGCID tiene para centralizar toda la información (OCDE-AGCID, 20I3)—, sí poseen una clara validez para disponer de un perfil de Chile como proveedor de cooperación. Así, a partir de la combinación de esta fuente básica con la información recogida por la SEGIB en sus informes de cooperación Sur-Sur, el presente epígrafe trata de presentar los principales rasgos que caracterizan a la cooperación realizada por Chile.

En este sentido, si se atiende a la evolución del total de fondos gestionados por la AGCID, se aprecian importantes oscilaciones, que van desde los 4,58 millones de dólares ejecutados en
2006 al poco más del millón de euros desembolsado en 20II, pasando por los 7,3I millones de dólares ejecutados en 2009 (gráfico I) $^{12}$. Como es lógico, el trágico terremoto sufrido por el país en febrero de 2010 parece estar en la base del repliegue experimentado por los fondos de la cooperación chilena en esos años. No obstante, algunas estimaciones realizadas por la OCDE que tratan de aglutinar el conjunto de la cooperación chilena, y no solo de la AGCID, sitúan en 42,2 millones de dólares los fondos desembolsados en 20I2, de los que el 74\% sería canalizado a través de organismos multilaterales (OCDE-AGCI, 2OI3).

11 Este epígrafe es una ampliación y actualización de lo recogido en Santander, G. (20I3).

12 Además de los problemas de fragmentación de la información señalados, varias razones aconsejan leer con cautela estos datos. Algunas oscilaciones pueden deberse a diferencias metodológicas en la forma de computar la información, especialmente en lo referido a la actividad de los fondos de cooperación - como el de Chile-México- o al Programa de Becas, lo que hace complicado realizar comparaciones rigurosas entre años. 


\section{GRÁFICO I}

Evolución de los fondos de cooperación gestionados por la AGCID

(millones de dólares)

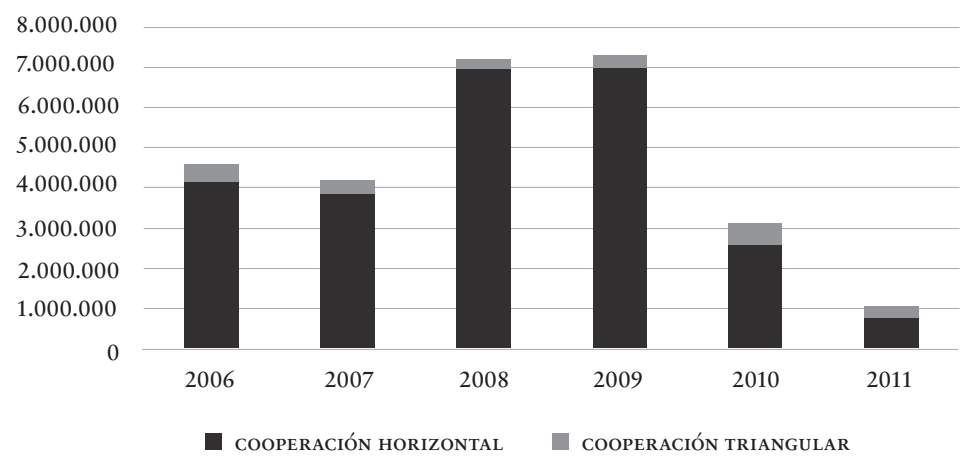

Fuente: Elaboración propia a partir de AGCID (20IOa, 20I0b, 20I0c, 20IIa, 20IIb y 20IIc)

Atendiendo a la información recogida por el último informe de cooperación Sur-Sur de la SEGIB - y basado, por tanto, en el número de proyectos y acciones ejecutadas y no en la cuantía de dichas intervenciones-Chile ocupa el cuarto lugar, tras México, Brasil y Argentina, entre los países oferentes de cooperación Sur-Sur horizontal en la región. Este país habría actuado como oferente en 56 proyectos y 44 acciones en 20I3, lo que supone el ro,93\% de los proyectos totales desarrollados en la región (SEGIB, 20I5). Mientras, en lo que se refiere a proyectos de cooperación triangular, Chile aparece de manera muy destacada como el principal oferente de la región, por delante de los tres países anteriormente citados y siendo responsable del 39,7\% de los proyectos triangulares desarrollados en la región en 2013 . Al analizar cada una de estas dos modalidades — bi- lateral y triangular - se dedican los siguientes apartados.

\section{La cooperación bilateral de Chile ${ }^{13}$}

En lo que se refiere a la cooperación bilateral de Chile y respecto de los principales países destinatarios de los fondos ejecutados por la AGCID en 20IO-20II (gráfico 2), México ocupa un lugar destacado desde que en 2006 se crease el Fondo Conjunto de Cooperación Chile-México que, como se detalla más adelante, tiene como principal objetivo fortalecer la cooperación entre ambos países en el sector público y dispone de un presupuesto anual de dos millones de dólares (AGCID, 20IOa).

13 Se utiliza el término "bilateral" — con el que aquí se alude solo a la cooperación que Chile realiza - en lugar de "horizontal", como suele utilizar la propia cooperación chilena. 
Guillermo Santander Campos

Chile como oferente de cooperación Sur-Sur: la progresiva adaptación a los nuevos tiempos y responsabilidades

\section{GRÁFICO 2}

Principales receptores de la cooperación bilateral gestionada por la AGCID, 2010-2011 (millones de dólares)

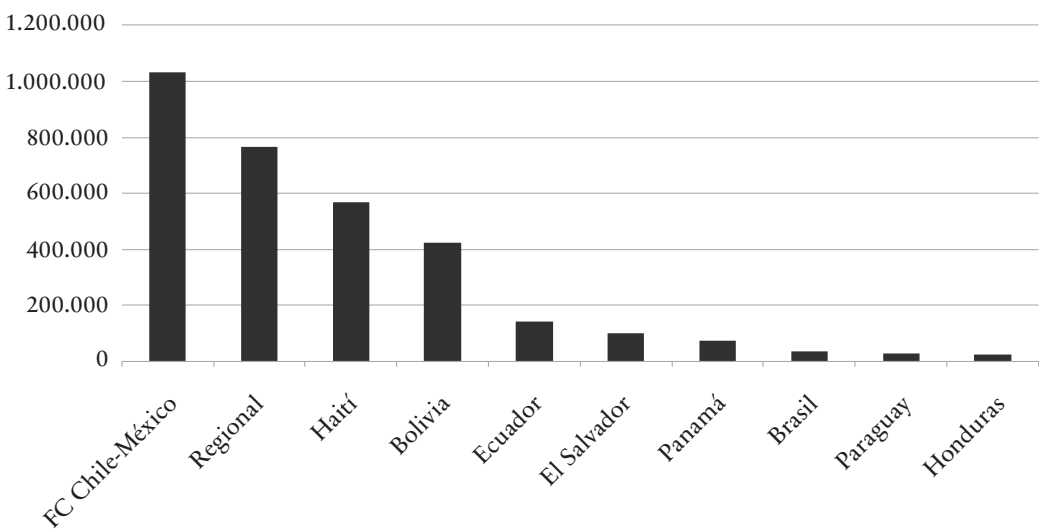

Fuente: Elaboración propia a partir de AGCID (20IOb y 20IIb)

Tras México, y al margen de las acciones de impacto regional no asignables a ningún país determinado, Haití y Bolivia aparecen como los destinos prioritarios de los fondos gestionados por la AGCID. Otros como Ecuador, El Salvador, Panamá, Brasil, Paraguay y Honduras, aparecen a continuación a considerable distancia.

Por su parte, los informes de la SEGIB, atendiendo al número de proyectos y no a la cuantía económica de los mismos, señala a Chile como un país con un considerable grado de concentración geográfica de su cooperación, de tal forma que en torno a la mitad de sus intervenciones suelen concentrarse en sus tres principales socios receptores. No obstante, en parte varían los países que conforman dicho ranking: mientras en 20II eran Bolivia y Panamá los que acompañaban a
México entre los principales receptores de la cooperación chilena, en 2012 lo hacían Ecuador y Uruguay (SEGIB, 2012 y 20I4).

Por otra parte, en lo que se refiere a los sectores a los que preferentemente se dirigen los fondos bilaterales gestionados por la AGCID, las tareas relacionadas con la superación de la pobreza, la protección y el desarrollo social concentran el 8I,6\% de los recursos, frente al $14,8 \%$ dirigido al fortalecimiento institucional y la modernización del Estado o el 3,5\% que se enmarcó en el ámbito del fomento productivo, la innovación y la competitividad (gráfico 3). De manera más concreta, son los sectores de cultura (23,15\%), superación de la pobreza $(20,56 \%)$ y desarrollo social (16,79\%), los que concentran la mayor parte de los recursos gestionados por la AGCID. Esta orientación marca- 
damente social de la cooperación chilena es destacada por los informes de la SEGIB, que señala que en 20 II Chile orientó la mayor parte de sus proyectos a la dimensión social, concediendo un menor espacio a la dimensión económica. La transferencia de capacidades en áreas educativas y sanitarias, el apoyo a los sistemas de protección y la asistencia social y el fortalecimiento de las instituciones públicas, aparecen como los ámbitos clave de trabajo de la cooperación chilena (SEGIB, 20I2). No obstante, otro informe más reciente de la SEGIB muestra que, aunque la mayor parte de los proyectos de cooperación chilena siguen orientados al ámbito social, otras dimensiones económicas —fundamentalmente la referida a los sectores productivos - parecen estar ganando peso (SEGIB, 20I4).
Dentro de este perfil, varias son las experiencias de la cooperación bilateral chilena que parecen haber alcanzado mayor relevancia. Una de ellas es, sin duda, la puesta en marcha del ya mencionado Fondo Conjunto de Cooperación Chile-México que, creado en el marco del Acuerdo de Asociación Estratégica firmado por ambos países en 2006 , no se puso efectivamente en marcha hasta dos años después. Desde entonces ha impulsado más de 40 proyectos de cooperación, desembolsando más de ocho millones de dólares. Este fondo, dirigido al intercambio de conocimientos en el ámbito público, en las áreas técnico-científica y educativo-cultural, parece estar promoviendo la horizontalidad y la apropiación en las relaciones establecidas entre ambos países, a la vez que está

\section{Gráfico 3}

Distribución sectorial de la cooperación bilateral gestionada por la AGCID (2010-2011)

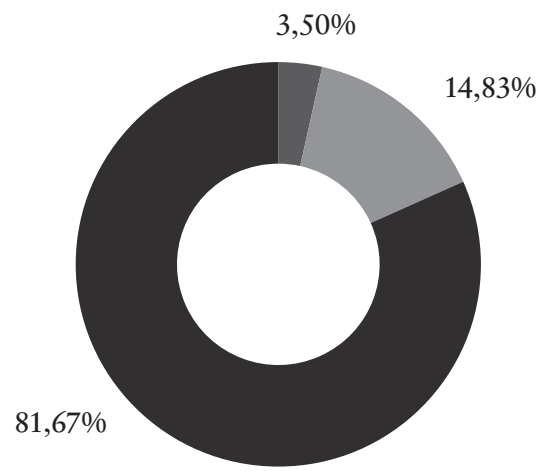

Fomento productivo, innovación y competitividad

Fortalecimiento institucional y modernización del Estado

- Superación de la pobreza, protección y desarrollo social

Fuente: Elaboración propia a partir de AGCID (20I0b y 20IIb) 
contribuyendo "a enfrentar de manera eficaz algunos desafíos del desarrollo, además de haber generado beneficios mutuos y soluciones locales [...]" (Sánchez y Sauterón, 20II: 6).

Otra experiencia destacada por diversos informes es la desarrollada entre Chile y Bolivia en el ámbito de la salud materno-infantil. A través de esta iniciativa, los técnicos hospitalarios chilenos capacitaron a personal médico boliviano, a la vez que se ha llevado a cabo una serie de transferencias de conocimientos en materia tecnológica y administrativa, con las que se ha logrado mejorar el diagnóstico y tratamiento ofrecido a los niños y niñas hospitalizados (SEGIB, 2OII: 43). Y, entre las diversas iniciativas de cooperación horizontal desarrolladas por Chile, puede aludirse también al apoyo ofrecido a Ecuador en materia de Derechos Humanos para el fortalecimiento de la Subsecretaría de Coordinación de Rehabilitación Social del Ministerio de Justicia (SEGIB, 20II: 45) o el emprendido en Haití en 2008, que tiene como finalidad apoyar a la pequeña infancia a través de la realización de transferencias técnicas para el Modelo de Educación Preescolar Integral, por poner algunos ejemplos (AGCID y PNUD, 20I2a).

\section{La cooperación triangular de Chile}

Uno de los rasgos característicos de la cooperación Sur-Sur chilena es su pionera y activa participación en iniciativas de carácter triangular (OCDE-AGCI, 20I3; AGCID y PNUD, 20I2b y 20I2c), en las que a los dos países en desarrollo implicados se les suma un donante tradicional del Norte. Una participación que, además, ha destacado por su notable diversificación, encontrándose un amplio número de países entre los socios - tanto donantes como receptores- de su cooperación triangular (cuadro I). 


\section{Cuadro 1}

Socios de la cooperación triangular chilena (2010-2012)

\begin{tabular}{|c|c|c|c|c|c|c|c|c|c|c|}
\hline & \multicolumn{9}{|c|}{ SOCIOS DONANTES } \\
\hline & & 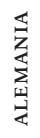 & 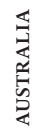 & 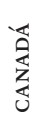 & $\begin{array}{l}\int \\
\sum_{0}^{\circ} \\
0 \\
0 \\
0\end{array}$ & 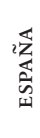 & 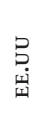 & 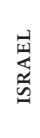 & $\begin{array}{l}z \\
\vdots \\
0 \\
\vdots \\
\vdots\end{array}$ & $\sum_{a}^{\mathbb{Z}}$ \\
\hline \multirow{13}{*}{ 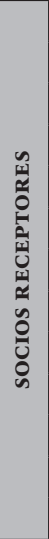 } & BOLIVIA & & & $x$ & & $x$ & & & $x$ & $x$ \\
\hline & COLOMBIA & $x$ & & & & & & & $x$ & \\
\hline & ECUADOR & & & & & & & & $x$ & $x$ \\
\hline & EL SALVADOR & $x$ & & & & $x$ & $x$ & & & \\
\hline & GUATEMALA & $x$ & & & & & & & & $x$ \\
\hline & HONDURAS & $\times$ & $\times$ & & & & & & & \\
\hline & MOZAMBIQUE & $x$ & & & & & & & & \\
\hline & NICARAGUA & $\times$ & & & & & & & & \\
\hline & PANAMÁ & & & & & & $x$ & & & \\
\hline & PARAGUAY & $x$ & $x$ & & & $x$ & $x$ & & $x$ & $x$ \\
\hline & R. DOMINICANA & $x$ & & & & & & & & $x$ \\
\hline & URUGUAY & $x$ & & & & & & & & \\
\hline & REGIONAL & $x$ & & & $x$ & & & $x$ & & \\
\hline
\end{tabular}

Fuente: Elaboración propia a partir de AGCID (20IOC y 20IIC) y SEGIB (20II, 2012 y 20I4).

De esta forma, tomando tan solo el bienio 20IO-20I2, se observa que Chile ha trabajado con hasta ocho "países fuente" (Alemania, Australia, Canadá, Corea del Sur, España, Estados Unidos, Israel y Japón) y el Programa Mundial de Alimentos (PMA), con los que ha logrado estar presente en nueve países del entorno latinoamericano (Bolivia, Colombia, Ecuador, El Salvador, Guatemala, Nicaragua, Paraguay, República Dominicana y Uruguay, además de los proyectos de alcance regional), y en un país de África Subsahariana (Mozambique).

No obstante, a pesar de dicha diversificación, existen importantes diferencias en la intensidad de trabajo mantenida con estos socios. Por un lado, en lo que se refiere a los países receptores, destaca de forma notoria Paraguay, que se erige como el principal destinatario de los fondos de cooperación triangular ejecutados por la AGCID en el período 20I0-20rI. Para el despliegue de su cooperación triangular en este país, Chile se ha asociado con hasta cinco donantes tradicionales diferentes (Alemania, Australia, España, Estados Unidos y Japón) y el Programa Mundial de Alimentos (PMA) de Naciones Unidas. Tras Paraguay, los proyectos triangulares de alcance regional y los dirigidos a Colombia, Bolivia, Guatemala y El Salvador, aparecen como los de mayor cuantía 
(AGCID, 20IOC y 20IIC). Por otro lado, en lo que se refiere a los países que actúan como donantes en la cooperación triangular chilena, Japón (25\%) y Alemania (I8,8\%) concentran el mayor número de proyectos (SEGIB, 20I2). No obstante, si se atiende al volumen de recursos aportados, es el segundo el que aparece, de forma nítida, como el principal socio donante de la cooperación triangular chilena en la actualidad - habiendo desembolsado 433.000 dólares en $20 \mathrm{II}$ - , seguido de Estados Unidos, Corea del Sur, Japón, España y Australia (AGCID, 20IIC) ${ }^{14}$.

Al igual como sucedía con la de carácter bilateral, la cooperación triangular ejecutada por AGCID se ha orientado de manera marcada al ámbito social, concentrando más del $60 \%$ de sus recursos en tareas relativas a la superación de la pobreza, la protección y el desarrollo social en el bienio 20I0-20II. No obstante, se observa una mayor atención dedicada al fortalecimiento institucional, ámbito al que la AGCID destinó casi la cuarta parte $(23,6 \%)$ de los fondos gestionados a través de esquemas triangulares (AGCID, 20IOC y
20IIc). Mientras, en lo que se refiere a la identificación de buenas prácticas en materia de cooperación, la propia AGCID, en conjunto con el PNUD, ha destacado dos proyectos. Por un lado, el llevado a cabo entre Alemania, Chile y República Dominicana, en el período 2007-2010, con el fin de promover el autoempleo en jóvenes rurales vulnerables, a partir de la transferencia de capacidades técnicas y de la aplicación de metodologías innovadoras en este campo. Y, por otro lado, el desarrollado desde 2010 entre Japón, Chile y Bolivia, en el ámbito de la rehabilitación inclusiva, a través del que se pretende formar a profesionales con el fin de responder a las necesidades existentes en esta área en el sistema público de salud boliviano (AGCID y PNUD, 20I2a). Otros proyectos triangulares como el realizado entre Chile, España y Paraguay, en el ámbito del fortalecimiento y modernización de la gestión pública, también han sido positivamente valorados (Alonso et al., 2OII; CAD-OCDE, 2OIO).

14 Si este análisis se extiende para el período 1998-20IO, el orden de principales países fuente por volumen de recursos aportados sería Japón, Suecia y Alemania (AGCID y PNUD, 20I2d). 


\section{El MODELO CHILENO DE COOPERACIÓN: NATURALEZA, RASGOS Y PRINCIPALES DESAFÍOS}

Si bien cabría hacer algunas distinciones y precisiones relevantes en función de los distintos períodos y gobiernos - lo que escapa a los propósitos del presente artículo-, la cooperación ofrecida por Chile ha presentado a lo largo de las últimas dos décadas unas líneas de continuidad que permiten componer un cierto modelo de cooperación Sur-Sur. A presentar y analizar tres de estos rasgos fundamentales y permanentes en el tiempo, se dedican los siguientes apartados.

\section{a) Un modelo de cooperación fuerte- mente asentado en las capacidades técnicas}

Un primer rasgo fundamental de la cooperación Sur-Sur de Chile es su marcado carácter técnico (Lazo, 20I2; Santander, 20II y 20I3). Esto no significa que Chile no lleve a cabo contribuciones de tipo financiero con algunos de sus socios en materia de cooperación internacional, pero sí que esta modalidad ocupa un lugar apenas significativo en comparación con el peso otorgado a la cooperación técnica (OCDE-AGCI, 20I3). Al margen de que, por su tamaño económico, Chile dispone de unos recursos financieros más limitados que otros países para dedicar a la cooperación internacional, este rasgo se relaciona con la percepción existente de que en las últimas décadas el país habría aplicado políticas exito- sas en determinados ámbitos ligados al desarrollo, obteniendo resultados positivos y acumulando un bagaje del que se pueden extraer enseñanzas útiles para aquellos países del entorno que afrontan retos similares en sus procesos de desarrollo.

Como resulta claro, esto último se relaciona estrechamente con la propia experiencia de desarrollo de Chile y el elevado nivel de desarrollo relativo alcanzado respecto de su región, de tal forma que este elemento acaba impregnando buena parte del modelo de cooperación chileno: es en los ámbitos en que se cosecharon éxitos a escala nacional donde más probable resulta que Chile acabe tratando de especializarse y compartir su experiencia a escala internacional. Así, como ilustran los informes elaborados por la AGCID y por SEGIB, y los diversos ejemplos de iniciativas exitosas que estos documentos frecuentemente recogen, el grueso de la cooperación Sur-Sur desplegada por Chile se asienta en la transferencia de conocimientos en aquellos ámbitos en los que, habiéndose detectado alguna problemática por parte de los países socios, Chile atesora experiencias y resultados que son positivamente valorados por su contraparte.

Esta concentración de la cooperación chilena en la transferencia de capacidades y conocimientos está en plena sintonía con los rasgos generales mostrados hasta la fecha por buena 
parte de la cooperación Sur-Sur, así como con algunas de las principales potencialidades que se le atribuyen a esta modalidad. No obstante, también está en la base de alguno de los principales retos a los que se enfrenta la cooperación chilena, de los que aquí cabe referirse a tres. En primer lugar, genera un desafío en términos de coordinación y articulación de actores. Como es lógico, las capacidades técnicas acumuladas por Chile, sobre las que descansa su modelo de cooperación Sur-Sur, se ubican en una amplia diversidad de ministerios y departamentos. Este hecho denota la versatilidad de las capacidades técnicas disponibles en Chile pero, en ocasiones, la ausencia de adecuados mecanismos de coordinación entre estos actores ha generado una cierta dispersión operativa y la ausencia de una concepción más integral del conjunto de las acciones desarrolladas (Martínez, 20I0). Un rasgo que, por lo demás, también ha impulsado una cooperación de carácter marcadamente intergubernamental - de gobierno a gobierno- (Lazo, 20I2), siendo hasta la fecha poco integradora de otros actores relevantes para los procesos de desarrollo, como la sociedad civil.

En segundo lugar, genera un desafío en términos de cuantificación de la cooperación realizada. Por un lado, porque, como expresión de la falta de articulación señalada, los diversos actores implicados en actividades de cooperación no reportan sobre ellas a ninguna entidad — como pudiera ser la
AGCID - que se encargue de centralizar y tratar estadísticamente esta información. Por otro lado, porque al ser un modelo que descansa en buena medida en el envío de funcionarios, se hace preciso establecer un sistema capaz de cuantificar económicamente esa dedicación y de computarla en los presupuestos de cooperación, tarea aún pendiente y actualmente en marcha.

En tercer lugar, genera un desafío en términos de evaluación de las acciones realizadas. En este sentido, a las problemáticas anteriores hay que sumar la ausencia de ejercicios que evalúen el impacto y los resultados alcanzados por las intervenciones realizadas. Si bien la evaluación y sistematización de las acciones llevadas a cabo debiera suponer siempre una tarea ineludible (Alonso, 20I4), parece serlo, aún en mayor grado, en un modelo de cooperación que pretende asentarse en el aprendizaje mutuo y en el intercambio de experiencias exitosas y que, por tanto, debiera hacer de la adecuada gestión del conocimiento - para la que la evaluación es indispensable- una de sus piedras angulares. En cualquier caso, sin obviar la importancia de los retos mencionados, son fundamentalmente señalados como expresión de un modelo de cooperación fuertemente asentado en la cooperación técnica, primer rasgo que quería ser destacado aquí. 


\section{b) Un modelo de cooperación poco sesgado en la selección de socios}

En estrecha relación con el rasgo anterior, cabe señalar que el hecho de que la cooperación chilena se asiente fundamentalmente en su propia experiencia de desarrollo y en la transferencia de los conocimientos acumulados en ese proceso, parece haber dado lugar a un modelo de cooperación Sur-Sur en que no se aprecian sesgos ideológicos a la hora de definir sus socios. Al hacer de la cooperación técnica y del intercambio de conocimientos su principal instrumento, la cooperación chilena parece estar más centralmente guiada por una suerte de cruce entre oferta y demanda - es decir, entre las capacidades disponibles por parte de Chile y los ámbitos en los que sus socios requieren cooperación-que por alianzas políticas previas o visiones ideológicas compartidas. Un rasgo que se aprecia con claridad si se observa el amplio y variado número de países y gobiernos con los que Chile coopera.

No obstante, este carácter más neutro que la cooperación chilena muestra a la hora de seleccionar a sus socios de trabajo, no quiere decir que no se detecten determinadas motivaciones de tipo político o ideológico en la cooperación Sur-Sur desplegada por este país. Las oscilaciones que ocasionalmente se han producido, en función de los distintos gobiernos, en la intensidad de las relaciones de cooperación mantenidas con países como Bolivia o Nicaragua o, por otro lado, los intereses geopolíticos que se derivan —en forma de votos y respaldo en foros internacionales- de la cooperación implementada con diversos países del ámbito caribeño, parecen claros ejemplos. Igualmente, conviene advertir que la menor permeabilidad que la cooperación chilena efectivamente muestra respecto de sesgos de tipo político e ideológico a la hora de seleccionar a sus socios, no debe confundirse con que este modelo no responda también a una visión política subyacente, cuyo despliegue tiene unas implicaciones y orientaciones determinadas.

Se trataría, en este sentido, de un modelo de cooperación que resulta funcional a su estrategia de inserción externa, a la imagen que pretende transmitir al exterior y a la búsqueda del posicionamiento internacional anteriormente descrito. En este sentido, Chile ha desarrollado una política exterior fuertemente vinculada a la apertura económica y comercial, tratando de posicionarse en el escenario internacional y en el contexto de la globalización como un país "abierto y flexible" y a hacer de ello una de sus principales señas de identidad. Es en el marco de esta matriz de política exterior en el que debe entenderse su opción de apostar por un modelo de cooperación Sur-Sur más técnico, menos ideológicamente marcado en la selección de sus socios y, por tanto, más abierto a una amplia diversidad de países. Una política exterior, en suma, que si bien se orienta a un determinado posicionamiento internacional 
y lleva implícita una visión política concreta - basada principalmente en nociones de carácter liberal-, en el ámbito estricto de su cooperación SurSur tiene su correlato en un modelo más aséptico desde el punto de vista político e ideológico.

Del mismo modo, cabe señalar que es precisamente la defensa de este modelo de cooperación abierto, "no excluyente" y especialmente centrado en el intercambio técnico, el que en buena medida explica también la entrada de Chile en la OCDE, de la que se deriva su participación como país observador en el CAD. Una pertenencia institucional que denota la existencia de "una visión compartida de cómo alcanzar el progreso (apertura al exterior y liberalización económica incluida) (...)" con los países occidentales y que ha llevado a que estos sitúen frecuentemente a Chile como un referente positivo dentro de los países en desarrollo y, muy concretamente, del ámbito latinoamericano (Gálvez, 20I2: I77; Riveros y Báez, 20I4). Un rasgo que sin duda también guarda relación con el especial protagonismo asumido por Chile en la puesta en práctica de nuevas modalidades como la cooperación triangular. Así, la apuesta por el modelo descrito no solo ha inducido a Chile a mostrarse especialmente activo e interesado respecto de esta nueva modalidad, sino que también le ha permitido presentarse ante los donantes tradicionales del CAD como un país responsable y un socio amable y confiable (Colacrai y Lorenzini, 2005) para la articulación de esquemas de cooperación triangular con ellos. Una dinámica de la que otros donantes emergentes latinoamericanos, con otras visiones y modelos de cooperación, se han mantenido más distanciados.

\section{c) Un modelo de cooperación de carácter reactivo}

Un tercer rasgo que parece caracterizar al modelo de cooperación chileno es su naturaleza fundamentalmente reactiva, en el sentido de que ha estado fuertemente orientado por la demanda. La existencia de una elevada solicitud de actividades de cooperación es, sin duda, reflejo de la positiva valoración que muchos países del entorno regional hacen de las políticas aplicadas por Chile en ámbitos muy diversos, fundamentalmente en lo relativo a políticas públicas y al fortalecimiento institucional en ámbitos sociales, la justicia, la seguridad ciudadana o el fomento del comercio (Lazo, 20I2). No obstante, ante la ausencia de una mayor articulación estratégica, este hecho también ha ido generando un modelo de cooperación un tanto impulsivo, que ha tratado de ir ofreciendo respuestas puntuales a las peticiones recibidas en función de las oportunidades y capacidades existentes, inhibiendo con ello el despliegue de una política con una lógica más proactiva.

Precisamente, la identificación de este comportamiento tradicionalmente reactivo en materia de cooperación - junto con otros factores como el ma- 
yor impulso y respaldo político del que actualmente parece gozar en Chile este ámbito de acción-, es uno de los elementos que está en la base de que se abriera un proceso de reflexión para la articulación de la denominada Política Chilena de Cooperación Internacional para el Desarrollo (AGCID, 20I5a), en cuyo marco se ha aprobado la primera Estrategia de Cooperación Internacional Chilena para el Desarrollo para el período 20I5-20I8. Un documento a través del cual, por vez primera, la cooperación chilena se marca tres objetivos estratégicos, tres áreas transversales de trabajo e identifica un conjunto de modalidades e instrumentos a desple- gar para lograr tales propósitos, lo que supone un avance sin precedentes en este sentido (AGCID, 20I5b) ${ }^{15}$.

En definitiva, la elevada cantidad y diversidad de ámbitos en los que Chile recibe solicitudes de cooperación técnica, junto con la ausencia de una mayor reflexión y articulación estratégica del conjunto de su sistema, ha conformado hasta la fecha un modelo de cooperación Sur-Sur de naturaleza fundamentalmente reactiva que, no obstante, parece estarse sometiendo a revisión para dotarlo de un carácter más estratégico y acorde con los retos que plantea la globalización.

\section{IDENTIDADES E INTERESES EN LA COOPERACIÓN CHILENA: LA ARTICULACIÓN DE UNA RESPUESTA ADAPTATIVA AL SISTEMA INTERNACIONAL}

Como se señaló en la introducción, la emergencia experimentada por la cooperación Sur-Sur en los últimos años se relaciona con los profundos cambios y transformaciones que se están produciendo en la estructura del sistema internacional. En el caso específico de la cooperación chilena, su irrupción no puede entenderse sin atender a determinados factores de tipo estructural, como el nivel de desarrollo relativo mostrado por el país o las dinámicas de liberalización e integración económicas que la globalización ha traído consigo. No obstante, una comprensión más completa de la cooperación Sur-Sur de Chile aconseja atender tam- bién una serie de elementos de carácter socio-cognitivo - referidos a las percepciones, identidades e intereses en juego-que habrían incidido en el surgimiento y fisonomía adoptada por esta política. Se trata de un conjunto

15 Los tres objetivos estratégicos que se plantean en este documento son: I) avanzar hacia un desarrollo inclusivo y sostenible; 2) fortalecer las asociaciones para un desarrollo compartido, y 3) consolidar el sistema nacional de cooperación internacional para el desarrollo. A la vez, las tres áreas transversales de trabajo son: I) igualdad de género y derechos de las mujeres; 2) innovación y uso de nuevas tecnologías para el desarrollo, y 3) fortalecimiento de capacidades y formación. 
de elementos que, por su naturaleza, aparecen en permanente estado de construcción y redefinición, así como en constante interacción con los cambios estructurales señalados. De este modo, junto con la existencia de una innegable voluntad de canalizar la solidaridad y promover el desarrollo en otros países de similar o menor nivel de renta, cabe destacar la concurrencia de tres procesos de tipo socio-cognitivo que habrían estimulado el creciente protagonismo desempeñado por Chile en las últimas dos décadas en su rol de proveedor de cooperación en el sistema internacional.

En primer lugar, existe un proceso de diferenciación, a través del cual Chile comenzó a autopercibirse como un país con unas capacidades y demandas singulares y diferenciadas de las existentes en un entorno regional que también se observaba como crecientemente heterogéneo. Un hecho que afectaría a su propia identidad, a la forma de concebir el sistema internacional y a la consideración del papel a desempeñar en su seno (Colacrai y Lorenzini, 2005). Elementos como el mayor grado de desarrollo mostrado en relación a su entorno regional o el elevado número de solicitudes de cooperación recibidas - tanto por parte de los países de su entorno, como por parte de los donantes tradicionales interesados en articular conjuntamente esquemas triangulares- sirvieron para reforzar esta percepción. Así, a partir de la contrastación de que se disponía de unas capacidades diferencia- das - atesoradas a partir de su propia experiencia de desarrollo y demandadas por otros países-, Chile comenzó a poner en marcha esquemas de cooperación técnica a través de las que fue dando forma a su cooperación Sur-Sur (Lazo, 20I2).

En segundo lugar, se ha producido un proceso de identificación positiva, en la medida en que desde los años noventa Chile fue progresivamente asumiendo e integrando en su discurso, que en un mundo globalizado e interdependiente resulta ineludible acudir a marcos de acción conjunta y establecer relaciones cooperativas con otros países, para alcanzar buena parte de los objetivos que dice perseguir. Su creciente compromiso financiero y su permanente e inequívoco respaldo a los organismos multilaterales, como vehículo a través del cual dotar de mayores niveles de gobernanza al sistema internacional, sería un claro ejemplo al respecto. Fruto de esta visión, Chile ha participado activamente en la MINUSTAH, ha formado parte activa de iniciativas como el mecanismo internacional lanzado por UNITAID en 2006 para la compra de medicamentos, así como acordó con el pNud la consolidación de la cooperación Sur-Sur que Chile realiza con otros países (PNUD, 2010), lo que desembocó en la puesta en marcha del Fondo Chile contra el Hambre y la Pobreza (AGCID-PNud, 20I4). Y en ese mismo sentido puede interpretarse la mencionada elaboración de su primera estrategia de cooperación que, entre otros propósitos, 
pretende contribuir a definir el rol y las responsabilidades que Chile debe asumir como actor internacional en un mundo globalizado. Una percepción que denota la activación de un proceso de identificación positiva con su entorno y con los actores que en él confluyen y que, por tanto, también habría servido de estímulo a su actividad como proveedor de cooperación en el sistema internacional.

Por último, cabe observar un proceso de instrumentalización, en la medida en que entre los factores que estimularon la promoción de actividades de cooperación Sur-Sur por parte de Chile, también se encontrarían las oportunidades que — se comenzó a percibiresta política puede atesorar para lograr otros objetivos de interés nacional. Se trata de un elemento que en el caso de Chile - como sucede con cualquier proveedor de cooperación, aunque con diversas intensidades - convive con las lógicas más estrictamente asociadas a la solidaridad y a la promoción del desarrollo de los países que reciben la cooperación. Esta instrumentalización de la política de cooperación para la persecución de objetivos nacionales se observa en, al menos, dos grandes niveles: la búsqueda de apoyos puntuales en los organismos internacionales por parte de los países socios y, de una manera más difusa, pero crucial para comprender el modelo chileno de cooperación, la funcionalidad que el despliegue de la política de cooperación tiene para presentarse a la comunidad internacional como un país abierto y responsable, en clara sintonía con su estrategia de inserción externa.

En suma, la conjunción de estos tres elementos socio-cognitivos, con el peso especialmente significativo que adquiere lo relativo al proceso de diferenciación que se deriva de su propia experiencia de desarrollo, es la que acaba finalmente componiendo, para el caso chileno, un modelo de cooperación Sur-Sur con un perfil marcadamente técnico y fuertemente asentado en las capacidades y conocimientos acumulados por el país. Un modelo que, además de ser de clara relevancia para promover el desarrollo de los países con los que coopera, acaba resultando funcional a la búsqueda de la inserción internacional que Chile persigue, contribuyendo así a conformar una respuesta adaptativa al sistema internacional vigente.

\section{Conclusiones}

Con el fin de la dictadura de Pinochet y el retorno de la democracia en 1990 , se generó en Chile un contexto político y social favorable al desarrollo de acciones de cooperación hacia los paí- ses de su entorno regional. Este hecho, junto con factores como el progreso y nivel de desarrollo relativo alcanzado por el país, propiciaron que desde entonces hasta la actualidad Chile haya 
ido asumiendo de manera creciente un rol dual en el sistema de cooperación internacional. Así, sin renunciar a seguir recibiendo flujos de ayuda y cooperación en determinados ámbitos, ha ido desempeñando un papel cada vez más relevante y significativo como proveedor de cooperación, destacando como uno de los principales protagonistas de la cooperación Sur-Sur en América Latina en los últimos años.

En este contexto, en las últimas dos décadas Chile ha ido conformando un modelo de cooperación Sur-Sur que debe entenderse en el marco de su política exterior y que ha presentado tres rasgos fundamentales: la concentración en actividades de tipo técnico, a través de la transferencia de capacidades y conocimientos; la escasa presencia de factores políticos e ideológicos en la selección de sus socios, que resultan numerosos y diversos, y su carácter fundamentalmente reactivo - aunque en actual estado de revisión-, en la medida en que se ha desplegado fundamentalmente a partir de la articulación de respuestas puntuales a las demandas de cooperación recibidas. Un modelo que, con el propósito de canalizar las aportaciones que Chile podía realizar en la promoción del desarrollo de los países de su entorno regional, habría resultado, además, funcional al marco estratégico de la política exterior chi- lena en su pretensión de adaptarse al sistema internacional vigente.

Este creciente interés en ser parte activa de la cooperación Sur-Sur, junto con avances recientes tan relevantes como el hecho de haberse dotado, por vez primera, de documentos estratégicos que pretenden orientar la política de cooperación internacional para el desarrollo, desplegada por el país, denotan una progresiva asunción de responsabilidades de Chile en la gobernanza de un sistema internacional en claro proceso de cambio. Como es lógico, este hecho, en plena sintonía con los nuevos roles y lógicas que este sistema parece reclamar, también lleva aparejado un conjunto de desafíos que la cooperación chilena deberá afrontar paulatinamente en los próximos tiempos. La consolidación de un sistema nacional de cooperación que integre y coordine a los distintos actores que pueden concurrir en esta política, el desarrollo de un adecuado sistema de gestión de la información y del conocimiento a partir de las acciones realizadas o la articulación de su cooperación Sur-Sur con los más amplios procesos de integración regional existentes en América Latina, por poner algunos ejemplos, son algunos de ellos. Unos desafíos, en suma, inherentes a las nuevas responsabilidades que Chile parece estar dispuesto a asumir en el sistema internacional de cooperación. 


\section{BIBLIOGRAFÍA}

AGCID (2015a). Politica Chilena de Cooperación Internacional para el Desarrollo. Agencia Chilena de Cooperación Internacional para el Desarrollo, Ministerio de Relaciones Exteriores. Santiago. AGCID (2015b). Estrategia de Cooperación Internacional Chilena para el Desarrollo. Agencia Chilena de Cooperación Internacional para el Desarrollo, Ministerio de Relaciones Exteriores. Santiago. AGCID (20Ira). Balance de Gestión Integral 20II. Agencia Chilena de Cooperación Internacional. Santiago de Chile: AGCID.

AGCID (20IIb). Estudio de la cooperación horizontal chilena gestionada por AGCID 20II. Agencia Chilena de Cooperación Internacional. Santiago: AGCID.

AGCID (20IIc). Estudio de la cooperación triangular chilena gestionada por AGCID 20II. Agencia Chilena de Cooperación Internacional. Santiago: AGCID.

AGCID (20I0a). Balance de Gestión Integral 2006-20ro. Agencia Chilena de Cooperación Internacional. Santiago: AGCID.

AGCID (20Iob). Estudio de la cooperación borizontal chilena gestionada por AGCID 20I0. Agencia Chilena de Cooperación Internacional. Santiago: AGCID.

AGCID (20I0c). Estudio de la cooperación triangular chilena gestionada por AGCID 20ro. Agencia Chilena de Cooperación Internacional. Santiago: AGCID.

AGCID y PNUD (20I4). Fondo Chile Contra el Hambre y la Pobreza. Agencia de Cooperación Internacional de Chile-Pro- grama de Naciones Unidas Para el Desarrollo. Santiago: AGCID-PNUD. AGCID y PNUD (20I2a). Buenas prácticas en cooperación sur-sur triangular de Chile: análisis de casos. Programa de Naciones Unidas para el Desarrollo-Agencia Chilena de Cooperación Internacional. Santiago: AGCID-PNUD.

AGCID y PNUD (2012b). Buenas prácticas en cooperación Sur-Sur triangular de Chile: criterios y metodología de selección de casos. Programa de Naciones Unidas para el Desarrollo-Agencia Chilena de Cooperación Internacional. Santiago: AGCID-PNUD.

AGCID y PNUD (2012c). La cooperación triangular en América Latina y Chile; 2006-2010. Tendencias, actores y prioridades. Programa de Naciones Unidas para el Desarrollo-Agencia Chilena de Cooperación Internacional. Santiago de Chile: AGCID-PNUD.

AGCID y PNUD (2012d). La cooperación triangular de Chile 1998-2010; características, fondos e instrumentos. Programa de Naciones Unidas para el Desarrollo-Agencia Chilena de Cooperación Internacional. Santiago: AGCID-PNUD.

Alonso, J. A. (20I4). "Eficacia agregada de la ayuda: incidencia sobre el crecimiento del receptor". Revista de Evaluación de Programas y Políticas Públicas no3. Madrid: Universidad Nacional a Distancia (UNED). pp. 154-I88.

Alonso, J.A.; Glennie, J. y Sumner, A. (20I4). "Recipients and Contributors: middle income countries and the futu- 


\section{Guillermo Santander Campos}

Chile como oferente de cooperación Sur-Sur: la progresiva adaptación a los nuevos tiempos y responsabilidades

re of development cooperation". DESA Working Paper no 135. Nueva York: Naciones Unidas.

Alonso, J.A.; Aguirre, P. y Santander, G. (20II). La cooperación triangular española en América Latina: un análisis de dos experiencias de interés. Fundación Carolina-CEALCI, DT no5I. Madrid: Fundación Carolina.

Aranda, G. y Riquelme, J. (20II). "La política exterior de Chile desde 1990. Inserción internacional y prioridad regional". Cuadernos sobre Relaciones Internacionales, Regionalismo y Desarrollo, vol.6, no II, enero-junio 20II, pp. II-4I. Briceño, J. (20I4). "Del regionalismo abierto al regionalismo post hegemónico en América Latina", en Soto, W. Política internacional e integración regional comparada en América Latina. FLACSO, pp. 23-34.

CAD-OCDE (20I0). La cooperación SurSur en el contexto de la eficacia de la ayuda. no Historias de caso de socios en cooperación Sur-Sur y triangular. Grupo de Tarea sobre Cooperación Sur-Sur, CAD-OCDE: París.

Colacrai, M. y Lorenzini, M.E. (2005). "La política exterior de Chile: jexcepcionalidad o continuidad? Una lectura combinada de 'fuerzas profundas' y tendencias". Confines no $1 / 2$, agosto-diciembre 2005, pp. 45-63.

Fermandois, J. (20I2). "De país problemático a nación preferida", en Artaza, M. y Ross, C. La política exterior de Chile, 1990-2009. Del aislamiento a la integración global. Santiago: RIL, pp. 47I-499.

Fermandois, J. (2006). “Inserción global y malestar regional: la política exterior chilena en el ciclo democrático, I9902006". Revista Estudios Internacionales vol. 39, noI54, julio-septiembre 2006, Universidad de Chile-IEI, Santiago. pp. 9I-99.

Flisfisch, A. (20I2). "La política exterior chilena y América del Sur”, en Artaza, M. y Ross, C. La política exterior de Chile, 1990-2009. Del aislamiento a la integración global. Santiago: RIL, pp. 527-560.

Gálvez, E. (20I2). "Diplomacia multilateral de Chile post 1990", en Artaza, M. y Ross, C. La política exterior de Chile, 1990-2009. Del aislamiento a la integración global. Santiago: RIL, pp. I65-I86. Gobierno de Chile (20I4). Programa de Gobierno de Michelle Bachelet 20I420I8. Santiago.

Gobierno de Chile (2010). Programa de Gobierno 2010-20I4, Santiago.

Lazo, C. (20I2). "Chile y la cooperación internacional: 20 años de experiencia (1990-2010), en Artaza, M. y Ross, C. La política exterior de Chile, 1990-2009. Del aislamiento a la integración global. Santiago: RIL, pp. 367-396.

Martínez, I. (2010). "La cooperación internacional en Chile", en Freres, Ch. (Dir.); Martínez, I. y Angulo, O. (20II). La inserción de América Latina en el cambiante sistema internacional de cooperación. Documento de Trabajo no46, Madrid: Fundación Carolina. Milet, P. (20I2). "La política exterior de Chile: enfoque histórico, 1990-2010", en Artaza, M. y Ross, C. La política exterior de Chile, 1990-2009. Del aislamiento a la integración global. Santiago: RIL, pp. 315-332. 
OCDE-AGCID (2013). Special Review of Chile. OCDE- Agencia Chilena de Cooperación Internacional.

PNUD (20I4). Informe de Desarrollo Humano. Sostener el progreso humano: reducir vulnerabilidades y construir resiliencia. Programa de Naciones Unidas para el Desarrollo. Naciones Unidas.

PNUD (20I0). "Programa País para Chile 20II-20I4". Programa de Naciones Unidas para el Desarrollo.

Prado, J.P. (20I4). "La Alianza del Pacífico: integración vía comercio y cooperación Sur-Sur", en Ayllón, B., T, Ojeda y J. Surasky (coords.). Cooperación SurSur. Regionalismo e integración en América Latina. Madrid: La Catarata-IUDC, pp. I46-I62.

Quezada, A. (20I2). "Relaciones de Chile con Centroamérica y El Caribe; pasado, presente y los nuevos desafíos”, en Artaza, M. y Ross, C. La politica exterior de Chile, 1990-2009. Del aislamiento a la integración global. Santiago: RIL, pp. 50I-526.

Riveros, L. y Báez, G. (20I4). "Chile y la oCDE. La dicotomía entre lo macroeconómico y el desarrollo humano". Revista Estudios Internacionales, vol. 46, noI79, septiembre-diciembre 2014. Universidad de Chile-IEI, Santiago. pp. 9-34.

Ross, C. (20I2). "Los desafíos de la política exterior chilena," en Artaza, M. y Ross, C. La politica exterior de Chile, 1990-2009. Del aislamiento a la integración global. Santiago: RIL, pp. 853-880.

Sanahuja, J.A. (2013). "Las nuevas geografías de la pobreza y la desigualdad y las metas de desarrollo global post-20I5", en Mesa, M. (coord.). El reto de la democracia en un mundo en cambio: respuestas políticas y sociales. Anuario CEIPAZ 2013-20I4. Madrid: CEIPAZ, pp. 6I-I00.

Sanahuja, J.A. (2009). "Del «regionalismo abierto» al «regionalismo post-liberal». Crisis y cambio en la integración regional en América Latina”, en Martínez, L.; Peña, L. y Vázquez, M. (coords.). Anuario de la Integración de América Latina y el Gran Caribe no 7, 2008-2009. Pp. II-54.

Sanahuja, J.A. (2008), “ ¿Un mundo unipolar, multipolar o apolar? La naturaleza y la distribución del poder en la sociedad internacional contemporánea", en VVAA, Cursos de Derecho Internacional de VitoriaGasteiz 2007. Universidad del País Vasco. pp. 297-384.

Sánchez, G. y Sauterón, C. (20II). Acuerdo de Asociación Estratégica entre los Estados Unidos Mexicanos y la República de Chile. El caso del Fondo Conjunto de Cooperación México-Chile. Instituto Mora-Task Team on South-South Cooperation.

Santander, G. (2016). La cooperación SurSur: entre la promoción del desarrollo y la funcionalidad política: los casos de Chile, Venezuela y Brasil. Tesis Doctoral en Ciencias Políticas y Relaciones Internacionales. Madrid: Universidad Complutense.

Santander, G. (20I3). "La cooperación chilena: una realidad en tránsito”, en Ayllón, B. y T. Ojeda (Coords.) (2013). La cooperación Sur-Sur y triangular en América 


\section{Guillermo Santander Campos}

Chile como oferente de cooperación Sur-Sur: la progresiva adaptación a los nuevos tiempos y responsabilidades

Latina. Políticas afirmativas y prácticas transformadoras. Madrid: IUDC-La Catarata, 87-III.

Santander, G. (20II). "Chile: explorando nuevos roles", en Santander, G. (Coord.). Nuevos donantes y cooperación Sur-Sur: estudios de caso. Estudios e Informes ICEI no 6. Madrid: Instituto Complutense de Estudios Internacionales-UCM.

SEGIB (20I5). Informe de la Cooperación Sur-Sur en Iberoamérica 2015. Secretaría General Iberoamericana.

SEGIB (20I4). Informe de la Cooperación Sur-Sur en Iberoamérica 20I3-20I4. Secretaría General Iberoamericana.

SEGIB (20I2). Informe de la Cooperación Sur-Sur en Iberoamérica 20I2. Secretaría General Iberoamericana.
SEGIB (20II). Informe de la Cooperación Sur-Sur en Iberoamérica 20II. Secretaría General Iberoamericana.

SEGIB (20IO). Informe de la Cooperación Sur-Sur en Iberoamérica 20I0. Secretaría General Iberoamericana.

SEGIB (2009). Informe de la Cooperación Sur-Sur en Iberoamérica 2009. Secretaría General Iberoamericana.

SEGIB (2008). Informe de la Cooperación Sur-Sur en Iberoamérica 2008. Secretaría General Iberoamericana.

Van Klaveren, A. (1998). "Inserción internacional de Chile”, en Tolosa, C. y Lahera, E. (eds). Chile en los noventa. Santiago: Presidencia de la República, pp.II7-I60. 